\title{
Emerging Agents for the Management of Nephrotic Syndrome: Progress to Date
}

\author{
Keisha L. Gibson ${ }^{1}$ - Panupong Hansrivijit ${ }^{2}$ Maria E. Ferris ${ }^{1}$
}

Published online: 8 December 2015

\begin{abstract}
Nephrotic Syndrome is a rare condition associated with high morbidity in the $20-40 \%$ of children and adolescents who fail to respond to standard immunosuppressive therapies. Novel non-immunologic mechanisms of widely used immunosuppressive therapies, as well as emerging anti-inflammatory drugs, and anti-fibrotics may play a crucial role in the treatment of patients with refractory disease. This article will review some of these treatments and their various stages of investigation.
\end{abstract}

\section{Key Points}

Directed therapies based on specific molecular targets may replace treatment decisions based on histology and steroid responsiveness in nephrotic syndrome.

Non-immunologic mechanisms for nephrotic syndrome therapies are emerging as key in the role of palliating proteinuric disease.

\section{Introduction}

Idiopathic nephrotic syndrome affects $1-3$ per 100,000 incident patients less than 16 years of age annually $[1,2]$. Defined by the triad of proteinuria, hypoalbuminemia, and hypercholesterolemia, nephrotic syndrome in fact is a

Keisha L. Gibson

kgibson@med.unc.edu

1 University of North Carolina Kidney Center at Chapel Hill, 7005 Burnett Womack, Chapel Hill, NC 27599-7155, USA

2 Chulalongkorn University, Bangkok, Thailand symptom caused by several glomerular disorders that ultimately involve injury to the podocytes, the specialized epithelial cells of the glomerulus. Primary nephrotic syndrome can be categorized by histologic patterns-minimal change disease (MCD), focal segmental glomerulosclerosis (FSGS), or membranous glomerulopathy-but clinicians most often categorize nephrotic syndrome by steroid responsiveness. The Kidney Disease Improving Global Outcomes (KDIGO) guidelines published in 2012 provide evidence-based recommendations for the treatment of children with steroid-sensitive and steroid-resistant nephrotic syndrome [3, 4]. Early studies from the International Study of Kidney Disease in Children (ISKDC) reported more than $80 \%$ of children with nephrotic syndrome have steroid-responsive disease with good long-term prognosis [5]. More contemporary studies suggest the incidence of steroid resistance may exceed $40 \%$ and is associated with poor long-term renal prognosis [6]. Unfortunately, 25-40\% of children and adolescents will be resistant to the standard therapies of not only steroids, but also cyclophosphamide, calcineurin inhibitors, and mycophenolate mofetil. The incidence of steroid-resistant disease is higher in African Americans, Hispanics, and older children ( $>12$ years of age) $[7,8]$. The need for novel treatments for this population is substantial. As emerging agents enter various stages of investigation, it will be important to consider the potential utility of these treatments in children with difficult-to-treat nephrotic syndrome (Table 1).

\section{Anti-Inflammatory Drugs}

Primary nephrotic syndromes are non-inflammatory conditions. The beneficial role of anti-inflammatory drugs like calcineurin inhibitors for these diseases extends beyond the 
inhibition of interleukin-1 (IL-1) signaling of T lymphocytes thought key in the development of increased glomerular capillary permeability. Interruption of the podocyte cytoskeleton ultimately leads to injury and flattening (effacement) of podocytes, interruption of the slit diaphragm and finally proteinuria. Synaptopodin stabilizes the actin cytoskeleton of the podocyte and is protected from degradation by phosphorylation. Calcineurin dephosphorylates synaptopodin, subjecting it to degradation by cathepsin L. This mechanism impedes calcineurin's ability to stabilize the cytoskeleton. Faul et al. [9] showed that the inhibition of calcineurin by cyclosporine A, protected against lipopolysaccharide-induced proteinuria in mice, and demonstrated that activation of calcineurin in the podocyte is sufficient to cause proteinuria by causing degradation of synaptopodin. While this example outlines a novel mechanism of an old drug, new insights on molecular targets are leading to novel drugs.

\subsection{Pentoxifylline}

Tumor necrosis factor (TNF)- $\alpha$ is a pro-inflammatory cytokine that has been demonstrated to be elevated in patients with nephrotic syndrome [10,11]. Pentoxifylline is a nonselective phosphodiesterase inhibitor and methylxanthine derivative similar to theophylline that possesses anti-inflammatory and immunomodulatory effects, and has been widely used to treat occlusive peripheral vascular disorders. Studies have demonstrated that pentoxifylline attenuates nephrotic syndrome secondary to membranous glomerulonephritis, lupus nephritis, and diabetic nephropathy [12-14]. The anti-proteinuric effect of pentoxifylline may be attributed to downregulation of TNF- $\alpha$ (Fig. 1). Other studies have demonstrated that pentoxifylline's anti-proteinuric effects may result from the decrease in urinary monocyte chemoattractant protein 1 (MCP-1), a chemokine that appears to be involved in the initiation and progression of tubulointerstitial damage in chronic kidney disease [15-17]. Chen et al. [15] reported a significant reduction of urinary protein/creatinine ratio in 17 adult patients with proteinuric glomerular disease (baseline vs. 6 months after pentoxifylline treatment, 2.82 vs. $1.79 \mathrm{~g}$ creatinine, $p<0.006$ ).

\section{2 p38 MAPK Signaling: Losmapimod}

The major families of MAPK (or mitogen-activated protein kinases) translate extracellular stimuli to intracellular responses. The p38 MAPK pathway has been identified as an important influence within several processes, including inflammation, differentiation, senescence, tumorogenesis, and apoptosis. Pro-apoptotic p38 MAPK signaling has been implicated in both experimental models of renal injury and in human glomerulopathies. P38 MAPK was found in podocytes and other glomerular cells after disease induction [18]. Inhibition of this signaling pathway was effective in reducing the severity of injury [19]. Podocyte p38 MAPK activation was increased in biopsy samples from adults with various forms of nephrotic syndrome [20]. These data provide support for the current multicenter, open-label, phase II, proof-of-mechanism study evaluating the reduction of proteinuria in individuals with corticosteroid or cyclosporine resistant FSGS, when treated with losmapimod, a p38 MAPK inhibitor (clinicaltrials.gov NCT02000440).

\section{Anti-Fibrotic Drugs}

\subsection{Endothelin Receptor Type 1A Antagonism: Sparsentan}

Endothelins (ETs) are potent regulators of arterial blood pressure. Endothelin-1 (ET-1), the only ET expressed in kidneys, plays a crucial role in cell growth/proliferation, fluid and electrolyte excretion, vascular tone, and immune functions. ET-type $\mathrm{A}_{1}\left(\mathrm{ETA}_{1}\right)$ receptors are located in vascular smooth muscle cells and the endothelial cells of arteries in the glomerulus, where they promote vasoconstriction and sodium retention along with cellular proliferation, inflammation, and fibrosis [21]. Signaling crosstalk between glomerular podocytes and endothelial cells has been identified as a factor in progressive glomerulosclerosis. SMAD proteins are condensed protein mediators of transcriptional activity. Various mechanisms of podocyte injury activate SMAD proteins associated with ET-1 precursor synthesis and ET-1 release. Paracrine ET-1/endothelin receptor type A (EDNRA) signaling causes mitochondrial oxidative damage and dysfunction in glomerular endothelial cells. Endothelial mitochondrial DNA damage and dysfunction-dependent paracrine activities are required for transforming growth factor beta-1 (TGF- $\beta 1$ )/SMAD-induced podocyte depletion and segmental glomerulosclerosis with nephrotic syndrome, elevated serum creatinine, and eventually decreased survival [22]. The mechanism underlying podocyte apoptosis after endothelial dysfunction has not been elucidated. Researchers have also demonstrated the ability to palliate podocyte effacement and proteinuria in mice with adriamycin-induced podocyte injury, by using sitaxsentan, a selective ETA receptor inhibitor [23]. A randomized, double-blind, activecontrol, dose-escalation study with a selective dual-acting receptor antagonist that has affinity for ETA $_{1}$ and angiotensin II receptors (type 1) is in progress. These investigators will evaluate the reduction of proteinuria in patients older than 8 years of age with biopsy-demonstrated FSGS (clinicaltrials.gov NCT01613118). 
Table 1 Trial data for emerging therapies in nephrotic syndrome

\begin{tabular}{llll}
\hline Drug & Target & Most current study & Includes children \\
\hline Pentoxifylline & TNF-alpha & Phase III & No \\
Losmapimod & p38 MAPK & Phase II & No \\
Sparsentan & Endothelin type A receptor & Phase II & Yes \\
Pirfenidone & TGF-beta & Phase II & No \\
Rituximab & CD20/ SMPDL-3b & Phase III & Yes \\
Abatacept & B7-1 & Pilot & Yes \\
\hline
\end{tabular}

The development of the therapies described in this article remains in various stages of investigation. Recognition of the importance of including children in these studies is growing

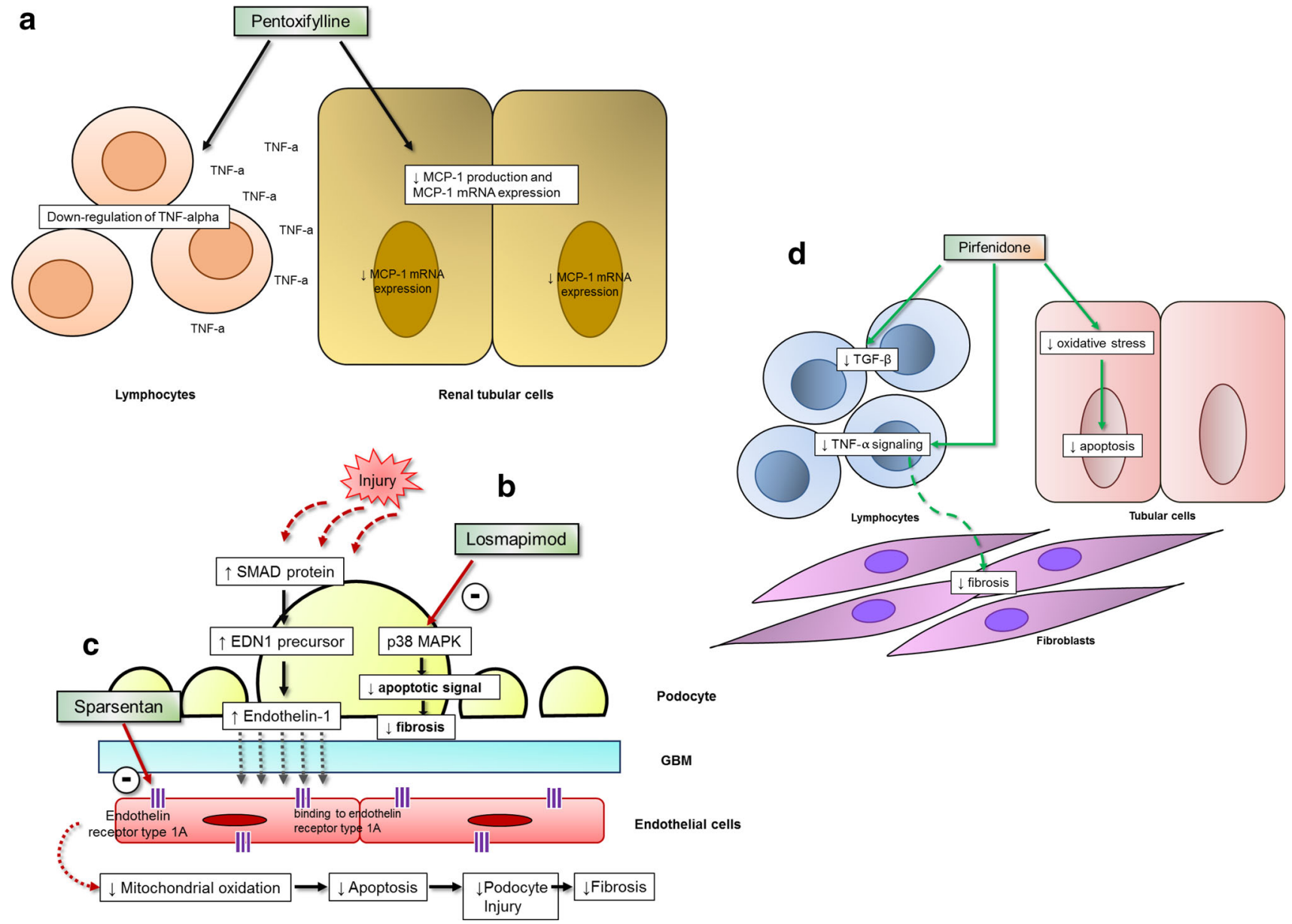

Fig. 1 Emerging therapies for nephrotic syndrome. a Pentoxifylline, a nonselective phosphodiesterase inhibitor, downregulates TNF- $\alpha$ release and reduces $\mathrm{MCP}-1$, thereby minimizing the inflammatory process. b Losmapimod inhibits pro-apoptotic activity of p38 MAPK in podocytes, resulting in decreased renal fibrosis. $\mathbf{c}$ TGF- $\beta 1$-induced expression of SMAD proteins stimulates podocyte endothelin-1 release. Endothelin-1 receptor activation stimulates endothelial cell mitochondrial oxidation damage, which, through unclear mechanisms, leads to further podocyte injury and renal fibrosis. Sparsentan,

\subsection{Pirfenidone}

Pirfenidone is a novel anti-fibrotic agent that has been shown to reduce TGF- $\beta 1$ production and block TNF-alpha production [24]. Pirfenidone may also have a role in an endothelin receptor type 1A antagonist inhibits this endothelial cell-podocyte cross signaling. d Pirfenidone reduces TGF- $\beta 1$ production, blocks TNF-alpha production, and reduces oxidative stress of renal tubular cells, making this drug a promising anti-fibrotic therapy for several glomerular disorders. EDN1 endothelin-1, GBM glomerular basement membrane, $M A P K$ mitogen-activated protein kinases, $M C P-1$ monocyte chemoattractant protein-1, $m R N A$ messenger RNA, $T G F-\beta 1$ transforming growth factor- $\beta 1, T N F$ tumor necrosis factor

reducing oxidative stress of renal tubular cells by inhibiting the mitochondrial apoptotic signaling pathway [25]. Glomerulosclerosis was effectively decreased in diabetic mice that were given pirfenidone, and in an animal model of subtotal nephrectomy, pirfenidone intake reduced TGF- 
$\beta$ production with consequently decreased tubulointerstitial fibrosis [26]. Clinical trials assessing the safety and efficacy of this therapy have been encouraging across multiple fibrotic conditions, including hepatitis $\mathrm{C}$-associated hepatitis, pulmonary fibrosis, and bladder dysfunction due to multiple sclerosis [27-30]. In an open-label study of 18 patients with FSGS treated with pirfenidone for a median of 13 months, the rate of glomerular filtration rate (GFR) decline was slowed by $25 \%$ from a baseline median of $-0.61 \mathrm{ml} / \mathrm{min}$ per $1.73 \mathrm{~m}^{2}$ to a median of $-0.45 \mathrm{ml} / \mathrm{min}$ per $\left.1.73 \mathrm{~m}^{2} ; p<0.01\right)$ [31]. Pirfenidone also improved the estimated GFR (eGFR) in a randomized, placebo-controlled trial of 77 patients with diabetic kidney disease [mean intergroup difference in eGFR change was $15.5 \mathrm{ml} /$ min per $1.73 \mathrm{~m}^{2}\left(-2.2 \mathrm{ml} / \mathrm{min}\right.$ per $1.73 \mathrm{~m}^{2}$ for placebo vs. $13.3 \mathrm{ml} / \mathrm{min}$ per $1.73 \mathrm{~m}^{2}$ for pirfenidone $1200 \mathrm{mg}, 95 \%$ confidence interval 1.1-9.9; $p=0.026)$ ] [32]. There were no significant differences among study groups in proteinuria change or urinary TGF- $\beta$ excretion from baseline to the end of study. Further studies to understand the potential clinical role of this therapy are needed.

\section{Biologic Agents}

\subsection{Rituximab}

As evidenced by the inclusion in the 2012 KDIGO guidelines, rituximab is quickly moving from being classified as an "emerging" therapy to standard secondary therapy for steroid-sensitive nephrotic syndrome [3]. The role of B-cell depletive therapy with rituximab, a chimeric anti-CD20 monoclonal antibody, is yet to be fully determined. A novel target for rituximab through podocyte sphingomyelinaselike phosphodiesterase $3 \mathrm{~b}$ protein (which stabilizes podocyte actin stress fibers and cell survival) has recently demonstrated an advantageous role for rituximab independent of B-cell depletion [33]. Various non-randomized studies have suggested a favorable response in some patients with idiopathic nephrotic syndrome. A recent randomized, double-blinded, placebo-controlled trial of 48 children with frequently relapsing steroid-sensitive or steroid-dependent disease showed a significant difference [267 vs. 101 relapse free days (Hazard Ratio 0.27, $p<0.0001)]$ for patients who received rituximab versus placebo, respectively [34]. Studies evaluating the efficacy of rituximab in patients with steroid- and/or calcineurin inhibitor-resistant disease have revealed mixed results.

\subsection{Abatacept}

Protein B7-1 (CD80) is commonly found on antigen presenting cells. This ligand functions as a co-stimulatory signal for $\mathrm{T}$ cells depending on which ligand it binds to. Binding with CD28 creates a stimulatory signal, while binding with cytotoxic $\mathrm{T}$ lymphocyte associated protein 4 (CTLA-4) promotes a regulatory signal. B7-1 is not expressed by normal podocytes, but has been found in human podocytes with various glomerulonephritides including FSGS and membranoproliferative glomerulonephritis (MPGN). Increased podocyte expression of B71 has additionally been found in mouse models of induced proteinuria [35, 36]. Yu et al. [36] found that B7-1 influences podocyte structure and function as increased podocyte migration, and proteinuria was associated with intact B7-1 expression in their in vitro studies. Their work further found that B7-1 bound to $\beta 1$ integrin (an essential ligand to bind podocytes to the glomerular basement membrane) can modify the actin cytoskeleton. Abatacept is a fusion protein of the Fc region of the immunoglobulin IgG1 and extracellular domain of CTLA-4. It blocks CD80 signaling through competitive binding for T-cell ligands, and has been proven safe and effective for patients with rheumatoid arthritis. Yu et al. were not only able to demonstrate in their in vitro studies that abatacept prevented B7-1-induced podocyte migration and prevented B7-1/ $\beta 1$ integrin association, they also showed they could induce proteinuric remission in four patients with recurrent FSGS post transplant who had failed on rituximab and another patient with steroid-resistant nephrotic syndrome. While the authors of this study concluded that B7-1 immunostaining of biopsies may identify a subgroup of patients who would benefit from treatment with abatacept, efforts to replicate these findings have not been widely successful [36]. Nonetheless, this emerging therapy provides an intriguing option for larger prospective clinical studies.

\section{Conclusions}

The need for novel therapies in nephrotic syndrome refractory to standard therapies is substantial. As our understanding of new molecular targets of both emerging and old therapies for nephrotic syndrome expand, it will be important to understand how to safely use these therapies in children and adolescents, and, therefore, we advocate for their inclusion in on-going clinical trials.

Acknowledgments This study does not include studies with human or animal subjects performed by any of the authors.

\section{Compliance with Ethical Standards}

Conflict of interest K. L. Gibson, P. Hansrivijit, and M. E. Ferris declare no conflicts of interest.

Funding No sources of funding were used to support the writing of this manuscript. 


\section{References}

1. McKinney PA, et al. Time trends and ethnic patterns of childhood nephrotic syndrome in Yorkshire, UK. Pediatr Nephrol. 2001;16(12):1040-4.

2. Uhlig $\mathrm{K}$, et al. Grading evidence and recommendations for clinical practice guidelines in nephrology. A position statement from Kidney Disease: improving Global Outcomes (KDIGO). Kidney Int. 2006;70(12):2058-65.

3. Lombel RM, et al. Treatment of steroid-sensitive nephrotic syndrome: new guidelines from KDIGO. Pediatr Nephrol. 2013; 28(3):415-26.

4. Lombel RM, et al. Treatment of steroid-resistant nephrotic syndrome in children: new guidelines from KDIGO. Pediatr Nephrol. 2013;28(3):409-14.

5. Nephrotic syndrome in children: prediction of histopathology from clinical and laboratory characteristics at time of diagnosis. A report of the International Study of Kidney Disease in Children. Kidney Int. 1978;13(2):159-65.

6. Kim JS, et al. High incidence of initial and late steroid resistance in childhood nephrotic syndrome. Kidney Int. 2005;68(3): 1275-81.

7. Bonilla-Felix M, et al. Changing patterns in the histopathology of idiopathic nephrotic syndrome in children. Kidney Int. 1999;55(5):1885-90.

8. Srivastava T, Simon SD, Alon US. High incidence of focal segmental glomerulosclerosis in nephrotic syndrome of childhood. Pediatr Nephrol. 1999;13(1):13-8.

9. Faul $\mathrm{C}$, et al. The actin cytoskeleton of kidney podocytes is a direct target of the antiproteinuric effect of cyclosporine A. Nat Med. 2008;14(9):931-8.

10. Bakr A, et al. Tumor necrosis factor-alpha production from mononuclear cells in nephrotic syndrome. Pediatr Nephrol. 2003;18(6):516-20.

11. Suranyi MG, et al. Elevated levels of tumor necrosis factor-alpha in the nephrotic syndrome in humans. Am J Kidney Dis. 1993;21(3):251-9.

12. Badri $\mathrm{S}$, et al. Effect of add-on pentoxifylline on proteinuria in membranous glomerulonephritis: a 6-month placebo-controlled trial. Clin Drug Investig. 2013;33(3):215-22.

13. Galindo-Rodriguez G, et al. Pentoxifylline in the treatment of refractory nephrotic syndrome secondary to lupus nephritis. J Rheumatol. 2003;30(11):2382-4.

14. Shan D, et al. Pentoxifylline for diabetic kidney disease. Cochrane Database Syst Rev. 2012;2:CD006800.

15. Chen YM, et al. Pentoxifylline ameliorates proteinuria through suppression of renal monocyte chemoattractant protein-1 in patients with proteinuric primary glomerular diseases. Kidney Int. 2006;69(8):1410-5.

16. Viedt C, Orth SR. Monocyte chemoattractant protein-1 (MCP-1) in the kidney: does it more than simply attract monocytes? Nephrol Dial Transplant. 2002;17(12):2043-7.

17. Vilayur E, Harris DC. Emerging therapies for chronic kidney disease: what is their role? Nat Rev Nephrol. 2009;5(7):375-83.

18. Stambe $C$, et al. Blockade of p38alpha MAPK ameliorates acute inflammatory renal injury in rat anti-GBM glomerulonephritis. J Am Soc Nephrol. 2003;14(2):338-51.
19. Sheryanna A, et al. Inhibition of p38 mitogen-activated protein kinase is effective in the treatment of experimental crescentic glomerulonephritis and suppresses monocyte chemoattractant protein-1 but not IL-1beta or IL-6. J Am Soc Nephrol. 2007;18(4):1167-79.

20. Koshikawa M, et al. Role of p38 mitogen-activated protein kinase activation in podocyte injury and proteinuria in experimental nephrotic syndrome. J Am Soc Nephrol. 2005;16(9):2690-701.

21. Jandeleit-Dahm KA, Watson AM. The endothelin system and endothelin receptor antagonists. Curr Opin Nephrol Hypertens. 2012;21(1):66-71.

22. Daehn I, et al. Endothelial mitochondrial oxidative stress determines podocyte depletion in segmental glomerulosclerosis. J Clin Invest. 2014;124(4):1608-21.

23. Buelli $\mathrm{S}$, et al. Beta-arrestin-1 drives endothelin-1-mediated podocyte activation and sustains renal injury. J Am Soc Nephrol. 2014;25(3):523-33.

24. Cain WC, et al. Inhibition of tumor necrosis factor and subsequent endotoxin shock by pirfenidone. Int J Immunopharmacol. 1998;20(12):685-95.

25. Chen JF, et al. Improved mitochondrial function underlies the protective effect of pirfenidone against tubulointerstitial fibrosis in 5/6 nephrectomized rats. PLoS One. 2013;8(12):e83593.

26. RamachandraRao SP, et al. Pirfenidone is renoprotective in diabetic kidney disease. J Am Soc Nephrol. 2009;20(8):1765-75.

27. Armendariz-Borunda $J$, et al. A pilot study in patients with established advanced liver fibrosis using pirfenidone. Gut. 2006;55(11):1663-5.

28. Azuma A, et al. Double-blind, placebo-controlled trial of pirfenidone in patients with idiopathic pulmonary fibrosis. Am J Respir Crit Care Med. 2005;171(9):1040-7.

29. Walker JE, Giri SN, Margolin SB. A double-blind, randomized, controlled study of oral pirfenidone for treatment of secondary progressive multiple sclerosis. Mult Scler. 2005;11(2):149-58.

30. Carter NJ. Pirfenidone: in idiopathic pulmonary fibrosis. Drugs. 2011;71(13):1721-32.

31. Cho ME, et al. Pirfenidone slows renal function decline in patients with focal segmental glomerulosclerosis. Clin J Am Soc Nephrol. 2007;2(5):906-13.

32. Sharma K, et al. Pirfenidone for diabetic nephropathy. J Am Soc Nephrol. 2011;22(6):1144-51.

33. Perosa F, et al. Generation of biologically active linear and cyclic peptides has revealed a unique fine specificity of rituximab and its possible cross-reactivity with acid sphingomyelinase-like phosphodiesterase 3b precursor. Blood. 2006;107(3):1070-7.

34. Ravani $\mathrm{P}$, et al. Rituximab is a safe and effective long-term treatment for children with steroid and calcineurin inhibitor-dependent idiopathic nephrotic syndrome. Kidney Int. 2013;84(5): 1025-33.

35. Reiser J, et al. Induction of B7-1 in podocytes is associated with nephrotic syndrome. J Clin Invest. 2004;113(10):1390-7.

36. Yu CC, et al. Abatacept in B7-1-positive proteinuric kidney disease. N Engl J Med. 2013;369(25):2416-23. 